\title{
The Very Cost Effective Graph Folding of the Join of Two Graphs
}

\section{El-Kholy EM ${ }^{1 *}$ and Mohamed HA ${ }^{2}$}

${ }^{1}$ Department of Mathematics, Faculty of Science, Tanta University, Tanta, Egypt

${ }^{2}$ Department of Mathematics, Faculty of Engineering at Shoubra, Banha University, Banha, Egypt

\begin{abstract}
In this paper, we studied the very cost effective graph property for the join graph of two graphs. In general this is may or may not be a very cost effective graph. We obtained the conditions for the join graph of two graphs to be a very cost effective graph. First we proved that the join graph $P_{n} \vee P_{m}$ of path graphs is very cost effective graph if $n+m$ is an even number and is not if $n+m$ is an odd number. Then we proved that the join graph of any two cycle graphs $C_{n}$ and $C_{m}$ where $n, m$ are both odd is very cost effective, and the join graph $P_{n} \vee C_{n}$ is a very cost effective graph if $n$ is an odd number. Also we proved that the join graph $G_{1} \vee G_{2}$ of two very cost effective graphs $G_{1}$ and $G_{2}$ is a very cost effective graph if $n\left(G_{1}\right)+n\left(G_{2}\right)$ is even. Finally we proved that the graph folding of the join graph of two very cost effective graphs not always very cost effective but this will be the case if the sum of the numbers of the vertices in the image of the graph folding is even.
\end{abstract}

Keywords: The join graph; Graph folding; Very cost effective graph

\section{Introduction}

A graph $G=(V, E)$ is a nonempty, finite set of elements called vertices together with a set of unordered pairs of distinct vertices of $\mathrm{G}$ called edges. The vertex set of $\mathrm{G}$ is denoted by $\mathrm{V}(\mathrm{G})$ and the edge set of $\mathrm{G}$ is denoted by $\mathrm{E}(\mathrm{G})$. A simple graph is a graph with no loops and no multiple edges. If $\mathrm{e}=\{u, v\}$ is an edge of a graph $\mathrm{G}$, then $u$ and $v$ are adjacent vertices, while $u$ and $e$ are incident [1]. Two adjacent vertices are called neighbors of each other. The degree (valency) of a vertex $v$ in a graph $\mathrm{G}$ is the number of edges incident to $v$ [2]. A vertex of degree 0 in $\mathrm{G}$ is called an isolated vertex [3]. A vertex $\mathrm{v}$ is said to be even or odd, according to whether its degree in $\mathrm{G}$ is even or odd [4]. If all the vertices of a graph $G$ have the same valency then it is called a regular graph [5]. The order of $G$, denoted $n(G)=|V(G)|$, is the number of vertices in $G$. The size of $G$, denoted $m(G)=|E(G)|$, is the number of edges in $\mathrm{G}$ [6]. A graph of order 1 is called a trivial graph, and a graph of order at least 2 is called a non-trivial graph. A graph of size 0 is called an empty graph. A nonempty graph has one or more edges [7]. A graph is said to be connected if every pair of vertices has a path connecting them [8], otherwise is called disconnected. For any vertex $v \in \mathrm{V}(\mathrm{G})$, the open neighborhood of $v$ is the set $\mathrm{N}(v)=\{u \in \mathrm{V}(\mathrm{G}) \mid u v \in \mathrm{E}(\mathrm{G})\}$, and the closed neighborhood of $v$ is the set $\mathrm{N}[v]=\{\mathrm{N}(v) \cup\{v\}\}$. For a set $\mathrm{S} \subseteq \mathrm{V}(\mathrm{G})$, its open neighborhood $\mathrm{N}(\mathrm{S})=\mathrm{U}_{v \in S} \mathrm{~N}(v)$, and its closed neighborhood is $\mathrm{N}[\mathrm{S}]=\mathrm{N}(\mathrm{S}) \cup \mathrm{S}$. A path $P n$ is graph in which any two vertices are connected by exactly one edge with two vertices of degree 1 , and the other $\mathrm{n}-2$ vertices of degree 2 .

\section{Definition (1.1)}

Let $G_{1}$ and $G_{2}$ be simple graphs and $f: G_{1} \rightarrow G_{2}$ be a continuous function. Then $\mathrm{f}$ is called a graph map, if

1. For each vertex $\mathrm{v} \in \mathrm{V}\left(\mathrm{G}_{1}\right)$, $\mathrm{f}(\mathrm{v})$ is a vertex in $\mathrm{V}\left(\mathrm{G}_{2}\right)$.

2. For each edge e $\epsilon \mathrm{E}\left(\mathrm{G}_{1}\right), \operatorname{dim}(\mathrm{f}(\mathrm{e})) \leq \operatorname{dim}(\mathrm{e})$, [4].

\section{Definition (1.2)}

A graph map $f: G_{1} \rightarrow G_{2}$ is called a graph folding if $f$ maps vertices to vertices and edges to edges, i.e., for each vertex $v \in V\left(G_{1}\right), f(v)$ is a vertex in $\mathrm{V}\left(\mathrm{G}_{2}\right)$ and for each edge e $\epsilon \mathrm{E}\left(\mathrm{G}_{1}\right), \mathrm{f}(\mathrm{e})$ is an edge in $\mathrm{E}\left(\mathrm{G}_{2}\right)[2]$.

Cost effective and very cost effective sets in graphs were introduced [6] and studied further [7]. Very cost effective bipartitions were also first introduced [6] and were motivated by the studies of unfriendly partitions [1].

\section{Definition (1.3)}

A vertex $v$ in a set $\mathrm{S}$ is said to be cost effective if it is adjacent to at least as many vertices in $\mathrm{V} \backslash \mathrm{S}$ as in $\mathrm{S}$, that is, $|\mathrm{N}(v) \cap \mathrm{S}| \leq|\mathrm{N}(v) \cap(\mathrm{V} \backslash \mathrm{S})|$. A vertex $v$ is very cost effective if it is adjacent to more vertices in $\mathrm{V} \backslash \mathrm{S}$ than in $\mathrm{S}$, that is, $|\mathrm{N}(v) \cap \mathrm{S}|<|\mathrm{N}(v) \cap(\mathrm{V} \backslash \mathrm{S})|$. A set $\mathrm{S}$ is (very) cost effective if every vertex $v \in S$ is (very) cost effective [6].

\section{Definition (1.4)}

A bipartition $\pi=\{S, V \backslash S\}$ is called cost effective if each of $S$ and $V \backslash S$ is cost effective, and $\pi$ is very cost effective if each of $S$ and $V \backslash S$ is very cost effective. Graphs that have a (very) cost effective bipartition are called (very) cost effective graphs [7].

Note that not every graph has a very cost effective bipartition, e.g., the cycle and the complete graphs of odd orders are not very cost effective.

\section{Definition (1.5)}

If $G_{1}$ and $G_{2}$ are vertex-disjoint graphs. Then the join, $G_{1} V G_{2}$, of $G_{1}$ and $G_{2}$ is a super graph of $G_{1}+G_{2}$, in which each vertex of $G_{1}$ is adjacent to every vertex of $G_{2}$. The vertex set $V\left(G_{1} V_{2}\right)=V_{1} \cup V_{2}$, [5].

\section{The Join Graph of Very Cost Effective Graph}

We will study the very cost effective graph property for the join graph of two graphs. First we pay attention to path graphs where any path graph is a very cost effective graph [6]. It should be noted that

*Corresponding author: El-Kholy EM, Department of Mathematics, Faculty of Science, Tanta University, Tanta, Egypt, Tel: 0403344352; E-mail: pro.entsarelkholy809@yahoo.com

Received October 11, 2017; Accepted November 15, 2017; Published November 21, 2017

Citation: El-Kholy EM, Mohamed HA (2017) The Very Cost Effective Graph Folding of the Join of Two Graphs. J Appl Computat Math 7: 375. doi: 10.4172/21689679.1000375

Copyright: () 2017 El-Kholy EM, et al. This is an open-access article distributed under the terms of the Creative Commons Attribution License, which permits unrestricted use, distribution, and reproduction in any medium, provided the original author and source are credited. 
Citation: El-Kholy EM, Mohamed HA (2017) The Very Cost Effective Graph Folding of the Join of Two Graphs. J Appl Computat Math 7: 375. doi: 10.4172/2168-9679.1000375

Page 2 of 6

the join graph $P_{n} \vee P_{m}$ is not necessary a very cost effective graph. For example $P_{2} \vee P_{3}$ and $P_{4} \vee P_{5}$ are not very cost effective graphs (Figure 1).

In general this is true if $\mathrm{n}+\mathrm{m}$ is an odd number, as it is proved in theorem (2.3). The following two lemmas give very cost bipartitions for path graphs $P_{n}$, where $n$ is odd or even.

\section{Lemma (2.1)}

Any path $P_{i}, i=1,2, \ldots, n$ where $n$ is even has the only very cost bipartition $\pi=\{S, V \backslash S\}$, where $S=\left\{v_{1}, v_{3}, \ldots, v_{n-1}\right\}$ and $V \backslash S=\left\{v_{2}, v_{4}, \ldots\right.$, $\left.v_{n}\right\}$. In this case $n(S)=n(V \backslash S)$.

\section{Proof:}

Let $u$ be a vertex in $S$, then $\mid N(u) \cap S)|=\varnothing| N,(u) \cap(V \backslash S) \mid=1$ or $2(1$ for an end vertex and 2 otherwise ) and hence $u$ is very cost vertex in $S$. Also, we can prove that $V \backslash S$ is a very cost effective set. Hence $\pi$ is a very cost bipartition (Figure 2a).

\section{Lemma (2.2)}

Any path $P_{i}, i=1,2, \ldots, n$ where $n$ is odd has the only very cost bipartitions $\pi_{1}=\left\{S_{1}, V_{1} \mid S_{1}\right\}$, where $S_{1}=\left\{v_{1}, v_{3}, \ldots, v n\right\}$ and $V_{1} \mid S_{1}=\left\{v_{2}\right.$, $\left.v_{4}, \ldots, v n_{-1}\right\}$ or $\pi_{2}=\left\{S_{2}, V_{2} \mid S_{2}\right\}$, where $S_{2}=\left\{v_{2}, v_{4}, \ldots, v n_{-1}\right\}, V_{2} \mid S_{2}=\left\{v_{1}\right.$, $\left.v_{3}, \ldots, v n\right\}$. In this case either $n\left(S_{1}\right)=n\left(V_{1} \mid S_{1}\right)+1$ or $n\left(V_{2} \mid S_{2}\right)=n\left(S_{2}\right)+1$.
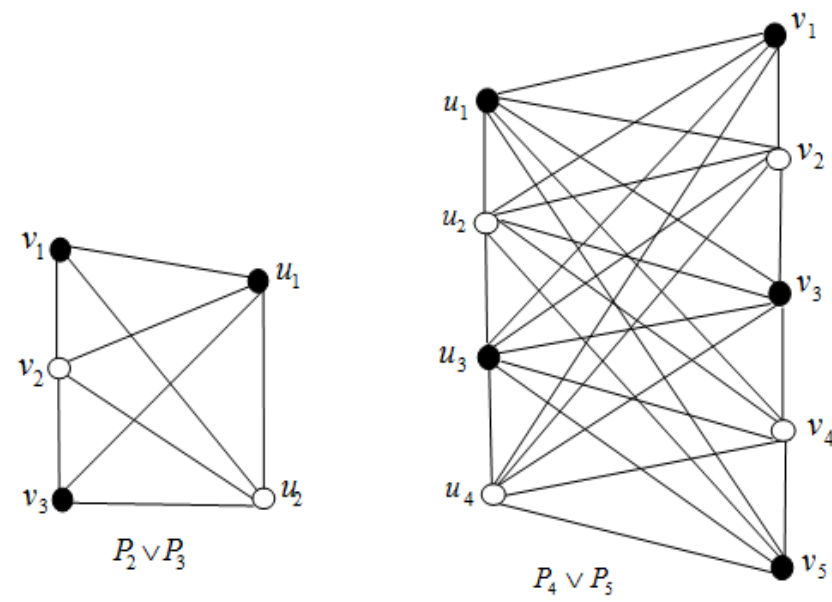

Figure 1: $P_{2} \vee P_{3}$ and $P_{4} \vee P_{5}$ cost effective graphs.

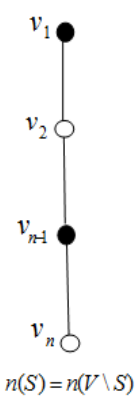

(a)

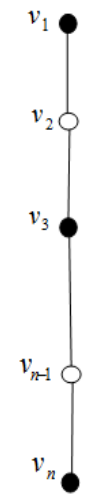

$n\left(S_{1}\right)=n\left(V_{1} \backslash S_{1}\right)+1$

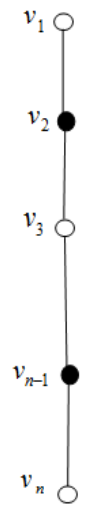

(b)

Figure 2: Bipartition cost effective graphs.

\section{Proof:}

By the same procedure as lemma (2.1), we can prove this lemma, Figure $2 b$.

\section{Theorem (2.3)}

The join of any path graphs $P_{n}$ and $P_{m}$ where $n+m$ is an odd number is not a very cost effective graph.

\section{Proof:}

Consider two path graphs $P_{n}$ and $P_{m}$ with very cost effective bipartitions $\pi_{1}=\left\{S_{1}, V_{1} \backslash S_{1}\right\}$ and $\pi_{2}=\left\{S_{2}, V_{2} \backslash S_{2}\right\}$, where $V_{1}=V\left(P_{n}\right)$ and $V_{2}=V\left(P_{m}\right)$. Without loss of generally, we may assume $\mathrm{n}$ is even and $\mathrm{m}$ is odd, then $n\left(S_{1}\right)=n\left(V_{1} \mid S_{1}\right)$ and let $n\left(S_{2}\right)=n\left(V_{2} \mid S_{2}\right)+1$.

Let $u$ be an end vertex in $S_{1}$, then $u$ is adjacent to more vertices of $V_{1} \backslash S_{1}$ than in $S_{1}$ by one. Now consider the join graph $P n \vee P_{m}$ with the bipartition $\pi=\{S, V \backslash S\}$ where $S=S_{1} \cup S_{2}$ and $V \backslash S=\left(V_{1} \mid S_{1}\right) \cup\left(V_{2} \backslash S_{2}\right)$, the vertex $u$ in this case will be adjacent to all the vertices of $P_{m}$ such that $n(N(u) \cap S)=n\left(S_{2}\right)$ and $n(N(u) \cap V \backslash S)=n\left(V_{2} \backslash S_{2}\right)$. But $n\left(S_{2}\right)=n\left(V_{2} \backslash\right.$ $\left.S_{2}\right)+1$. This mean that the vertex $u$ is adjacent to the same number of the vertices of $S_{2}$ and $V_{2} \mid S_{2}$, and thus the join graph $P_{n} \vee P_{m}$ is not a very cost effective graph.

\section{Theorem (2.4)}

The join of any path graphs $P_{n}$ and $P_{m}$ where $n+m$ an even number is a very cost effective graph.

\section{Proof:}

Consider two path graphs $P_{n}$ and $P_{m}$ with very cost effective bipartitions $\pi_{1}=\left\{S_{1}, V_{1} \mid S_{1}\right\}$ and $\pi_{2}^{n}=\left\{S_{2}, V_{2} \mid S_{2}\right\}$, respectively. We have two cases.

\section{Case 1: $n$ and $m$ are both even}

In this case $n\left(S_{1}\right)=n\left(V_{1} \mid S_{1}\right)$ and $n\left(S_{2}\right)=n\left(V_{2} S_{2}\right)$. For any vertex $u \epsilon$ $P n, u$ is adjacent to more vertices in $V_{1} \mid S_{1}$ than in $S_{1}$. But $\mathrm{u}$ in the join graph $P n \vee P_{m}$ will be adjacent to the same numbers of vertices of $S_{2}$ and $V_{2} \backslash S_{2}$, then $u$ is a very cost effective vertex. This also the case for any vertex $v \in P_{m}$. Then the join graph $P n \bigvee P_{m}$ is a very cost effective graph.

Case 2: $n$ and $m$ are both odd.

Consider the very cost effective bipartitions $\pi_{1}$ and $\pi_{2}$ where $n\left(S_{1}\right)=n\left(V_{1} \mid S_{1}\right)+1$ and $\left(V_{2} \mid S_{2}\right)=n\left(S_{2}\right)+1$. Let $u \in S_{1}$ so $u$ is adjacent to more vertices in $V_{1} \backslash S_{1}$ than in $S_{1}$ by at least one (1 or 2).

But in the join graph $P_{n} \vee P_{m}$, where $S=S_{1} \cup S_{2}$ and $V \backslash S=\left(V_{1} \mid S_{1}\right) \cup$ $\left(V_{2} \backslash S_{2}\right)$, the vertex $u$ will be adjacent to all the vertices of $V_{2} \backslash S_{2}$ and $S_{2}$, so $u$ is adjacent to more vertices of $V \backslash S$ than in $S$. Thus $u$ is very cost effective vertex. Now, let $v \in\left(V_{1} \mid S_{1}\right)$, so $v$ is adjacent to more vertices in $S_{1}$ than in $V_{1} \backslash S_{1}$ by two. But in the join graph $P_{n} \vee P_{m}$ the vertex $v$ will be adjacent to more vertices of $S$ than in $V \backslash S$, so $v$ is a very cost effective vertex, and consequently the join graph $P_{n} \vee P_{m}$ is a very cost effective graph.

\section{Theorem (2.5)}

The join graphs $P_{2} \vee P_{4}$ and $P_{3} \vee P_{5}$ are very cost effective graphs (Figure 3)

In the following we would like to answer the following questions

1. Is every join graph $G_{1} \vee G_{2}$ is a much cost?

2. If $G_{1}$ is very cost effective, is $G_{1} \vee G_{2}$ very cost effective for all graphs? 

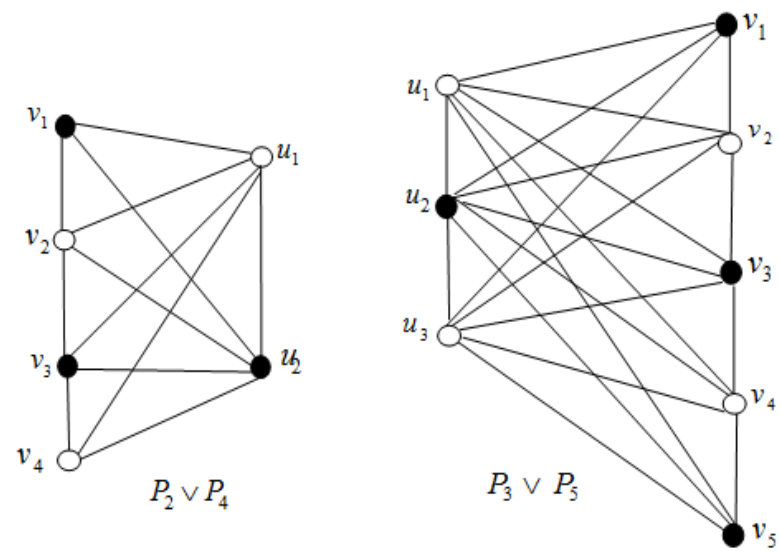

Figure 3: $P_{2} \vee P_{4}$ and $P_{3} \vee P_{5}$ are very cost effective graphs.
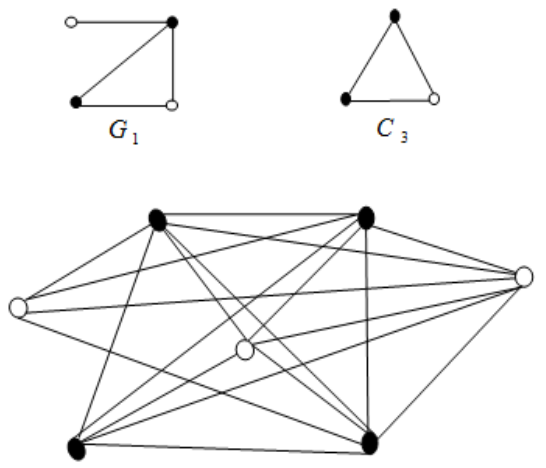

Figure 4: $G_{1} \vee C_{3}$ is not very cost effective.

3. If $G_{1}$ and $G_{2}$ are both very cost effective, is $G_{1} v G_{2}$ very cost effective?

The answer of the first question is, in general, no, i.e., the join $\mathrm{G}_{1}$ $v G_{2}$ of non-trivial connected graphs $G_{1}$ and $G_{2}$ may or may not a very cost effective graph. For example, the join graph $\mathrm{G}_{1} \vee \mathrm{C}_{3}$ shown in Figure 4 is not a very cost effective graph while the join graph $\mathrm{C}_{3} \mathrm{~V}_{3}$ shown in Figure 5 is a very cost effective graph.

The answer of the first question is yes for the following special case of graphs. Before discussing this case it should be noted that no cycle graph $\mathrm{C}_{2 \mathrm{n}+1}$ of odd order is very cost effective [6].

\section{Lemma (2.6)}

For a cycle graph $C n$, where $\mathrm{n}$ is odd the bipartition $\pi=\{S, V \backslash S\}$, where $S=\left\{v_{1}, v_{2}, v_{4}, \ldots, v n_{-1}\right\}$ and $V \backslash S=\left\{v_{3}, v_{5}, \ldots, v n\right\}$ is cost effective. In this case $n(S)=n(V \backslash S)+1$.

It is easy to prove the vertex $v_{1} \in S$ is cost effective while the other vertices in both $S$ and $V \backslash S$ are very cost effective vertices and hence $\pi$ is a cost effective bipartition (Figure 6).

Note that the bipartition $\pi=\{V \backslash S, S\}$ is also a cost bipartition.

\section{Theorem (2.7)}

The join graph of any two cycle graphs $C n$ and $C_{m}$ where $n, m$ are both odd is a very cost effective graph.

\section{Proof:}

Let $C n$ and $C_{m}$ be any two cycle graphs where $n, m$ are both odd.

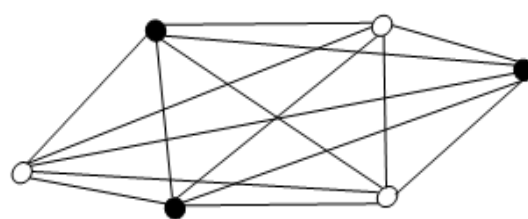

Figure 5: $\mathrm{C}_{3} \vee \mathrm{C}_{3}$ is a very cost effective.

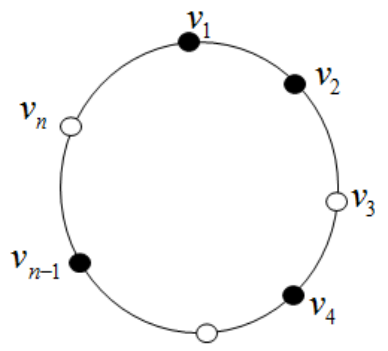

Figure 6: $v_{1} \in S$ is cost effective

Choose the cost effective bipartitions $\pi_{1=}\left\{S_{1}, V_{1} \mid S_{1}\right\}$ and $\pi_{2}=\left\{S_{2}, V_{2} \mid\right.$ $\left.S_{2}\right\}$ such that $n\left(S_{1}\right)=n\left(V_{1} \mid S_{1}\right)+1$ and $n\left(V_{2} \mid S_{2}\right)=n\left(S_{2}\right)+1$. Now consider the join graph $C_{n} \vee C_{m}$ with the cost bipartition $\pi=\{S, V \backslash S\}$, where $S=S_{1}$ $\cup S_{2}$ and $V \backslash S=\left(V_{1} \backslash S_{1}\right) \cup\left(V_{2} \backslash S_{2}\right)$. Now, let $u \in S_{1}$, if $u$ is a very cost effective vertex, then $u$ must be adjacent to more vertices of $V_{1} \mid S_{1}$ than in $S_{1}$ and since $u$ is adjacent to all the vertices of $S_{2}$ and $V_{2} \mid S_{2}$ and $n\left(S_{2}\right)=n\left(V_{2} \mid\right.$ $\left.S_{2}\right)-1$, then $u$ is still a very cost effective vertex in the join graph $C_{n} \vee C_{m}$. Now, suppose $u \in S_{1}$ is a cost effective vertex, then $u$ will be adjacent to at least as many vertices in $V_{1} \backslash S_{1}$ as in $S_{1}$ and since $u$ is adjacent to all the vertices of $S_{2}$ and $V_{2} \mid S_{2}$ and $n\left(V_{2} \mid S_{2}\right)=n\left(S_{2}\right)+1$. Thus $u$ is adjacent to more vertices in $S$ than in $V \backslash S$ by at least one and consequently $u$ is a very cost effective vertex. Now, let $u \in\left(V_{1} \mid S_{1}\right)$, then $u$ must be adjacent to more vertices in $S_{1}$ than in $V_{1} \mid S_{1}$ certainly by at least two and since $u$ is adjacent to all the vertices of $S_{2}$ and $\left(V_{2} \mid S_{2}\right)$ and $n\left(S_{2}\right)=n\left(V_{2} \mid S_{2}\right)-1$, then $\mathrm{u}$ is still a very cost effective vertex in the join graph $C_{n} \vee C_{m}$. Also, we can prove that any vertex of $S_{2}$ or $V_{2} \backslash S_{2}$ is a very cost effective vertex and hence $\pi$ is a very cost bipartition, i.e., $C_{n} \vee C_{m}$ is a very cost effective graph.

\section{Theorem (2.8)}

The join graph $C_{3} \vee C_{5}$ is a very cost effective graph (Figure 7).

Now let $G_{1}$ be a very cost effective graph and $G_{2}$ any non-trivial connected graph. The join graph of $G_{1} \vee G_{2}$ is not always very cost effective; the following example illustrates this fact.

\section{Theorem (2.9)}

The join graph $C_{3} \vee C_{4}$ shown in Figure $8 \mathrm{a}$ is not a very cost effective graph while the join graph $P_{3} \vee C_{3}$ is very cost effective (Figure 8b).

Thus the answer of the second question is, in general, no. The answer is yes in the following case.

\section{Theorem (2.10)}

The join graph of any path $P_{n}$ and cycle $C_{n}$ where $n$ is odd is a very cost effective graph.

Proof:

By the same procedure as in theorem (2.7) we can prove this theorem. 


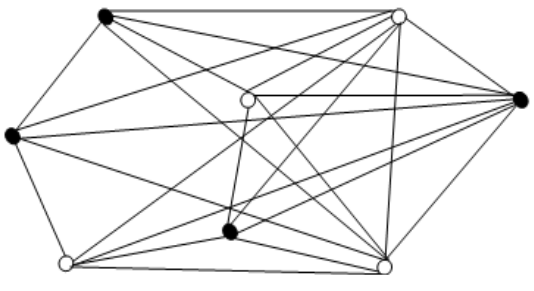

Figure $7: \mathrm{C}_{3} \vee \mathrm{C}_{3}$ is very cost effective.

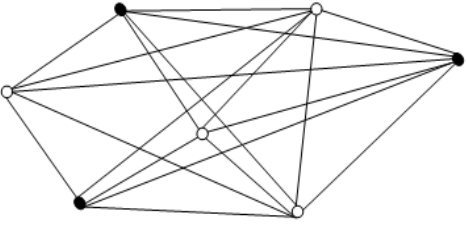

(a)

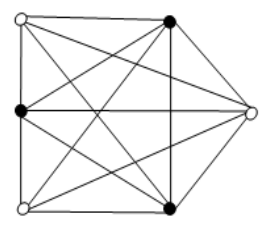

(b)
Figure 8: (a) $C_{3} \vee C_{4}$ is not cost effective; (b): $P_{3} \vee C_{3}$ is very cost effective.

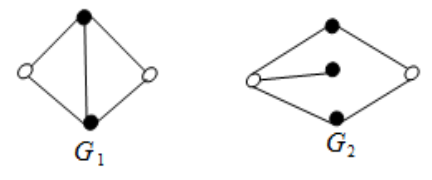

$G_{1}, G_{2}$ are very $\cos t$ effective graphs

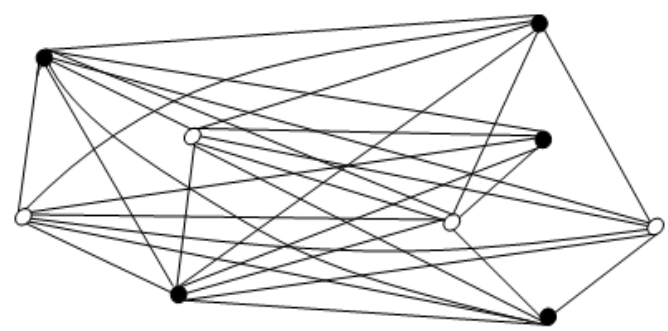

Figure 9: $G_{1} \vee G_{2}$ is not very cost effective.

Now, we come to the third question. The answer of the third question is also, in general, no, i.e., if $G_{1}$ and $G_{2}$ are two very cost effective graphs, then the join graph $G_{1} \vee G_{2}$ may or may not be very cost effective. For example, if $G_{1}$ and $G_{2}$ are the very cost effective graphs shown in Figure 9, then the join graph $G_{1} \vee G_{2}$ is not very cost effective.

While the join graph $K_{4} \mathrm{~V} K_{, 22}$ is a very cost effective graph (Figure 10).

The following theorem gives the condition for which the join graph of two very cost effective graphs is very cost effective.

\section{Theorem (2.11)}

The join graph of two non- trivial connected very cost effective graphs $G_{1}$ and $G_{2}$ is a very cost effective graph if $n\left(G_{1}\right)+n\left(G_{2}\right)$ is even.

\section{Proof:}

Let $G_{1}$ and $G_{2}$ be any non-trivial connected graphs, we have two cases

(1) $n\left(G_{1}\right)$ and $n\left(G_{2}\right)$ are both even. Then each graph has a very cost effective bipartition. Let $\pi_{1}=\left\{S_{1}, V_{1} \mid S_{1}\right\}$ and $\pi_{2}=\left\{S_{2}, V_{2} \mid S_{2}\right\}$, be such that

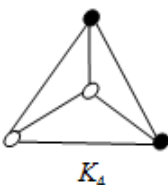

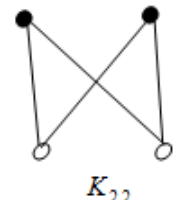

$K_{22}$
$K_{2}, K_{2,2}$ are very $\cos t$ effective graphs

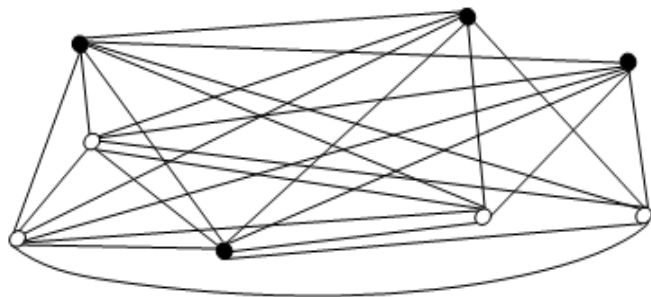

Figure 10: $K_{4} \vee K_{, 22}$ is a very cost effective graph.

$n\left(S_{1}\right)=n\left(V_{1} \mid S_{1}\right)$ and $n\left(S_{2}\right)=n\left(V_{2} \backslash S_{2}\right)$. Now consider the join graph $G_{1} \vee G_{2}$ with the cost bipartition $\pi=\{S, V \backslash S\}$, where $S=S_{1} \cup S_{2}$ and $V \backslash S=\left(V_{1} \backslash S_{1}\right)$ $\cup\left(V_{2} \backslash S_{2}\right)$. Let $u \in S_{1}$, then $u$ is adjacent to more vertices of $V_{1} \backslash S_{1}$ than in $S_{1}$ and since $u$ is adjacent to all the vertices of $S_{2}$ and $V_{2} \backslash S_{2}$, then $u$ is still a very cost effective vertex in the join graph $G_{1} \vee G_{2}$. Also we can prove that any vertex of $V_{1} \mid S_{1}$ or $S_{2}$ or $V_{2} \backslash S_{2}$ is a very cost effective vertex and hence $\pi$ is a very cost effective bipartition, i.e., $G_{1} \vee G_{2}$ is a very cost effective graph.

(2) $n\left(G_{1}\right)$ and $n\left(G_{2}\right)$ are both odd. Suppose that $\pi_{1}=\left\{S_{1}, V_{1} \backslash S_{1}\right\}$ and $\pi_{2}=\left\{S_{2}, V_{2} \backslash S_{2}\right\}$ are very cost effective bipartitions such that $n\left(S_{1}\right)=n\left(V_{1} \backslash\right.$ $\left.S_{1}\right)+1$ and $n\left(V_{2} \backslash S_{2}\right)=n\left(S_{2}\right)+1$. Let $\pi=\{S, V \backslash S\}$ be a cost bipartition of the join graph $G_{1} \vee G_{2}$, where $S=S_{1} \cup S_{2}$ and $V \backslash S=\left(V_{1} \mid S_{1}\right) \cup\left(V_{2} \backslash S_{2}\right)$. Now, let $u \in S_{1}$, then $u$ must be adjacent to more vertices of $V_{1} S_{1}$ than in $S_{1}$ and since $u$ is adjacent to all the vertices of $S_{2}$ and $V_{2} \mid S_{2}$ and $n\left(S_{2}\right)=n\left(V_{2} \backslash\right.$ $\left.S_{2}\right)-1$, then $u$ is still a very cost effective vertex in the join graph $G_{1}$ $\vee G_{2}$. Now, let $u \in V_{1} \mid S_{1}$, then $u$ must be adjacent to more vertices in $S_{1}$ than in $V_{1} \mid S_{1}$ certainly by at least two and since $u$ is adjacent to all the vertices of $S_{2}$ and $V_{2} \backslash S_{2}$, then $u$ is adjacent to more vertices in $S$ than in $V \backslash S$ by at least one and $u$ is a very cost effective vertex in the join graph $G_{1} \vee G_{2}$. Also we can prove that any vertex of $S_{2}$ or $V_{2} \mid S_{2}$ is very cost effective vertex and hence $\pi$ is a very cost effective bipartition, i.e., $G_{1}$ $V G_{2}$ is a very cost effective graph.

\section{Theorem (2.12)}

1) The join graph $C_{4} \vee W_{1,3}$ of the very cost effective graphs $C_{4}$ and $W_{1,3}$ is once again is a very cost effective graph (Figure 11).

2) Let $G_{1}$ and $G_{2}$ be the very cost effective graphs shown in Figure 12. Then the join graph $G_{1} \vee G_{2}$ is also very cost effective graph (Figure 12).

\section{The Join Graph Folding of Very Cost Effective Graphs}

In this section we study very cost effective graph folding of a new graph obtained by the join of two other graphs.

\section{Definition (3.1)}

Let $G_{1}=\left(V_{1}, E_{1}\right), G_{2}=\left(V_{2}, E_{2}\right), G_{3}=\left(V_{3}, E_{3}\right)$ and $G_{4}=\left(V_{4}, E_{4}\right)$ be graphs. Let $\mathrm{f}: \mathrm{G}_{1} \rightarrow \mathrm{G}_{3}$ and $\mathrm{g}: \mathrm{G}_{2} \rightarrow \mathrm{G}_{4}$ be graph maps. A join map $\mathrm{f}^{\mathrm{v}} \mathrm{g}$ : $\mathrm{G}_{1} \mathrm{VG}_{2} \rightarrow \mathrm{G}_{3} \mathrm{VG}_{4}$ is a map defined by

For each vertex $v \in V_{1} \cup V_{2},(f V g)(v)=\left\{\begin{array}{l}f(v), \text { if } v \in V_{2} \\ g(v), \text { if } v \in V_{2}\end{array}\right.$ 


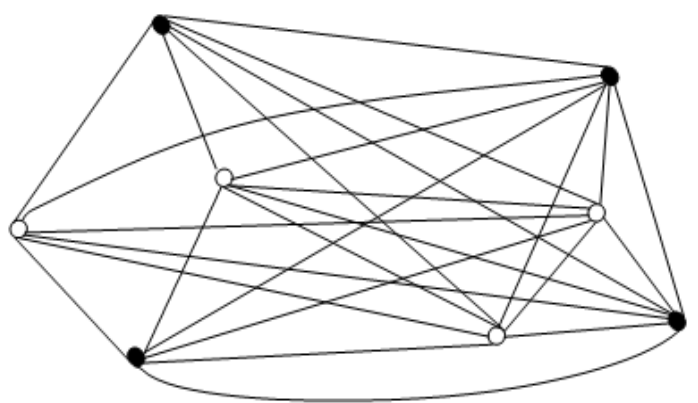

Figure 11: $C_{4} \vee W_{1,3}$ of the very cost effective
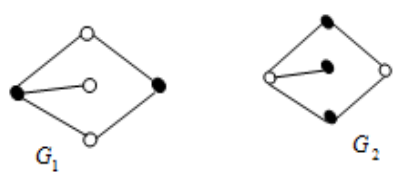

$G_{1}, G_{2}$ are very cost effective graphs

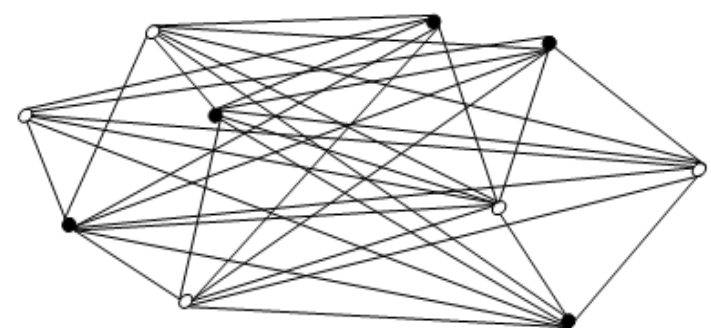

Figure 12: $G_{1} \vee G_{2}$ is also very cost effective graph.

For each edge $\mathrm{e}=\left(\mathrm{v}_{1}, \mathrm{v}_{2}\right), \mathrm{v}_{1} \in \mathrm{V}_{1}$ and $\mathrm{v}_{2} \in \mathrm{V}_{2},(\mathrm{fV} \mathrm{g})\{\mathrm{e}\}=\left\{\mathrm{f}\left(\mathrm{v}_{1}\right), \mathrm{g}\left(\mathrm{v}_{2}\right)\right\}$ $\in \mathrm{G}_{3} \mathrm{VG}_{4}$.

If $\mathrm{e}=\left(\mathrm{u}_{1}, \mathrm{v}_{1}\right) \in \mathrm{E}_{1}$ then $(\mathrm{f} \vee \mathrm{g})\{\mathrm{e}\}=\left\{\mathrm{f}\left(\mathrm{u}_{1}\right), \mathrm{f}\left(\mathrm{v}_{1}\right)\right\}$, also if $\mathrm{e}=\left(\mathrm{u}_{2}, \mathrm{v}_{2}\right) \in \mathrm{E}_{2}$, then $(\mathrm{fV} g)\{\mathrm{e}\}=(\mathrm{f} \bigvee \mathrm{g})\left\{\left(\mathrm{u}_{2,} \mathrm{v}_{2}\right)\right\}=\left\{\mathrm{g}\left(\mathrm{u}_{2}\right), \mathrm{g}\left(\mathrm{v}_{2}\right)\right\}[3]$.

\section{Definition (3.2)}

A graph folding f: $G_{1} \rightarrow G_{2}$ between two graphs $G_{1}$ and $G_{2}$ is a very cost effective graph folding if the image $f\left(G_{1}\right)=H \subseteq G_{2}$ is a very cost effective graph [8].

It should be noted that the image of a graph folding $f: G \rightarrow G$ of a very cost effective graph $\mathrm{G}$ may or may not be a very cost effective graph

e.g., if $\mathrm{G}$ is the cycle graph $\mathrm{C}_{4}$ where $\mathrm{V}\left(\mathrm{C}_{4}\right)=\left\{\mathrm{u}_{1}, \mathrm{u}_{2}, \mathrm{u}_{3}, \mathrm{u}_{4}\right\}$ and $\mathrm{E}\left(\mathrm{C}_{4}\right)=\left\{\mathrm{e}_{1}, \mathrm{e}_{2}, \mathrm{e}_{3}, \mathrm{e}_{4}\right\}$ then the graph folding $\mathrm{f}: \mathrm{C}_{4} \rightarrow \mathrm{C}_{4}$ defined by $\mathrm{f}\left\{\mathrm{u}_{1}\right.$, $\left.\mathrm{u}_{2}, \mathrm{u}_{3}, \mathrm{u}_{4}\right\}=\left\{\mathrm{u}_{3}, \mathrm{u}_{2}, \mathrm{u}_{3}, \mathrm{u}_{4}\right\}$ and $\mathrm{f}\left\{\mathrm{e}_{1}, \mathrm{e}_{2}, \mathrm{e}_{3}, \mathrm{e}_{4}\right\}=\left\{\mathrm{e}_{4}, \mathrm{e}_{3}, \mathrm{e}_{3}, \mathrm{e}_{4}\right\}$ is a very cost effective graph folding since the image is $\mathrm{P}_{3}$ (Figure 13).

From now on the omitted vertices or edges will be mapped into themselves. Also if $\mathrm{G}$ is the very cost effective graph shown in Figure 14 where $V(G)=\left\{v_{1}, v_{2}, v_{3}, v_{4}\right\}$ and $E(G)=\left\{e_{1}, e_{2}, e_{3}, e_{4}, e_{5}\right\}$ then the graph folding $g: G \rightarrow G$ defined by $g\left\{v_{1}\right\}=\left\{v_{3}\right\}$ and $g\left\{e_{1}, e_{2}\right\}=\left\{e_{4}, e_{3}\right\}$ is not a very cost effective.

Once again if $f$ and $g$ are both very cost effective graph foldings, the join map ( $\mathrm{f} \vee \mathrm{g}$ ) may or may not be a very cost effective graph folding. The following two theorems illustrate this.

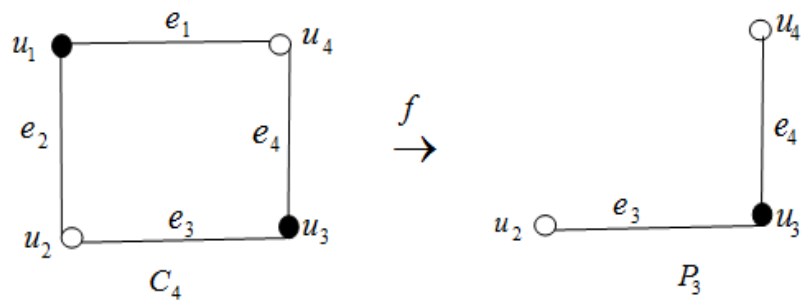

Figure 13: $P_{3}$ is also very cost effective graph.

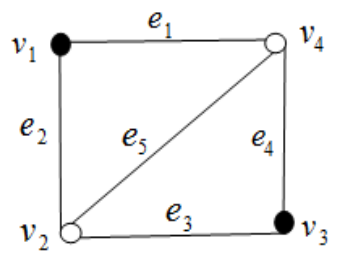

G

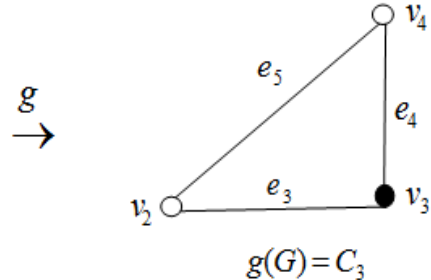

$g(G)=C_{3}$

Figure 14: $G$ is not cost effective graph
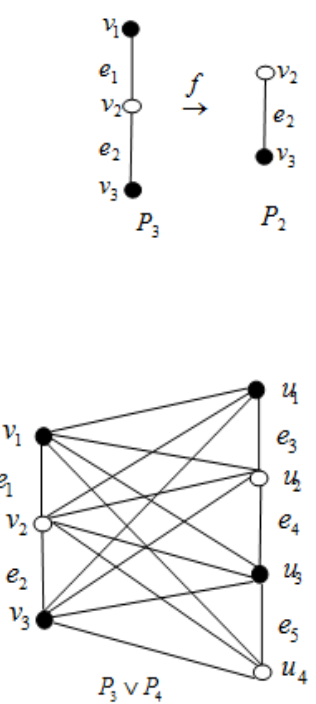

Figure 15: $f \vee g: G_{1} \vee G_{2} \rightarrow G_{1} \vee G_{2}$ is a very cost effective graph.

\section{Theorem (3.3)}

Let $G_{1}=P_{3}$ and $G_{2}=P_{4}$ as shown in Figure 15. Let $f: G_{1} \rightarrow G_{1}$ and g: $\mathrm{G}_{2} \rightarrow \mathrm{G}_{2}$ be the very cost effective graph foldings defined by $f\left\{\mathrm{v}_{1}\right\}=\left\{\mathrm{v}_{3}\right\}$, $\mathrm{f}\left\{\mathrm{e}_{1}\right\}=\left\{\mathrm{e}_{2}\right\}$ and $\mathrm{g}\left\{\mathrm{u}_{1}, \mathrm{u}_{4}\right\}=\left\{\mathrm{u}_{3}, \mathrm{u}_{2}\right\}$ and $\mathrm{g}\left\{\mathrm{e}_{3}, \mathrm{e}_{5}\right\}=\left\{\mathrm{e}_{4}, \mathrm{e}_{4}\right\}$. The join graph folding $\mathrm{f} V \mathrm{~g}: \mathrm{G}_{1} V_{\mathrm{G}_{2}} \rightarrow \mathrm{G}_{1} V_{\mathrm{G}_{2}}$ defined by $(\mathrm{f} \vee \mathrm{g})\left\{\mathrm{u}_{1}, \mathrm{u}_{4}, \mathrm{v}_{1}\right\}=\left\{\mathrm{u}_{3}, \mathrm{u}_{2}, \mathrm{v}_{3}\right\}$ is a very cost effective graph folding.

\section{Theorem (3.4)}

Let $G_{1}$ and $G_{2}$ be any connected graphs and $f$, $g$ are very cost effective graph foldings of $G_{1}$ and $G_{2}$, respectively. Then $(f \vee g)\left(G_{1} \vee G_{2}\right)$ is a very cost effective graph folding if no. $\mathrm{V}\left(\mathrm{f}\left(\mathrm{G}_{1}\right)\right)+$ no. $\mathrm{V}\left(\mathrm{g}\left(\mathrm{G}_{2}\right)\right)$ is even. The proof is almost the same as the proof of theorem (2.11). 


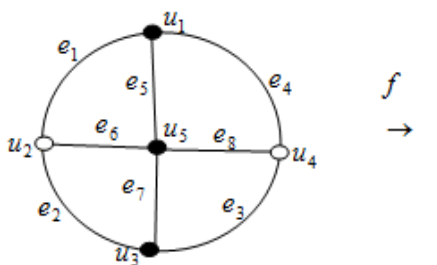

$W_{1,4}$

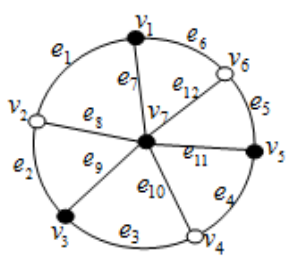

$W_{1,6}$

$$
\begin{gathered}
f \vee g \\
W_{1,4} \vee W_{1,6} \quad \rightarrow
\end{gathered}
$$
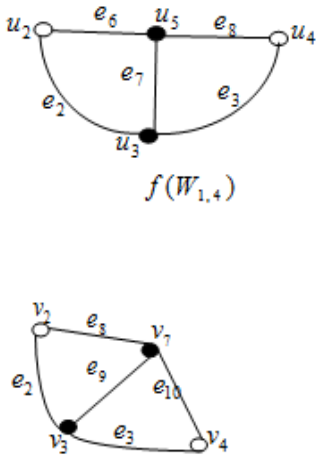

$g\left(W_{1,6}\right)$

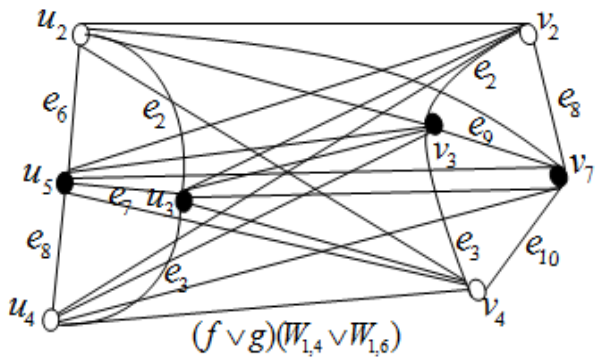

Figure 16: $f \vee g: W_{1,4} \vee W_{1,6} \rightarrow W_{1,4} \vee W_{1,6}$ is a very cost effective graph.

\section{Theorem (3.5)}

Consider the wheel graphs $\mathrm{W}_{1,4}$ and $\mathrm{W}_{1,6}$ shown in Figure 16. Let $\mathrm{f}: \mathrm{W}_{1,4} \rightarrow \mathrm{W}_{1,4}$ and $\mathrm{g:} \mathrm{W}_{1,6} \rightarrow \mathrm{W}_{1,6}$ be the very cost effective graph folding defined By $f\left\{u_{1}\right\}=\left\{u_{3}\right\}$ and $f\left\{e_{1}, e_{4}, e_{5}\right\}=\left\{e_{2}, e_{3}, e_{7}\right\}$ and $g\left\{v_{1}, v_{5}, v_{6}\right\}=\left\{v_{3}, v_{3}\right.$, $\left.\mathrm{v}_{4}\right\}$ and $\mathrm{g}\left\{\mathrm{e}_{1}, \mathrm{e}_{4}, \mathrm{e}_{5}, \mathrm{e}_{6}, \mathrm{e}_{7}, \mathrm{e}_{11}, \mathrm{e}_{12}\right\}=\left\{\mathrm{e}_{2}, \mathrm{e}_{3}, \mathrm{e}_{4}, \mathrm{e}_{3}, \mathrm{e}_{9}, \mathrm{e}_{9}, \mathrm{e}_{10}\right\}$. Then The join graph folding $\mathrm{f} \vee \mathrm{g}: \mathrm{W}_{1,4} \vee \mathrm{W}_{1,6} \rightarrow \mathrm{W}_{1,4} \vee \mathrm{W}_{1,6}$ is a very cost effective graph folding (Figure 16).

\section{References}

1. Aharoni R, Milner EC, Prikry K (1990) Unfriendly partitions of a graph. Journal of Combinatorial Theory, Series B 50: 1-10.
2. El-Kholy E, Al-Esawy A (2005) Graph folding of some special graphs. Journal of Mathematics and Statistics 1: 66-70.

3. El-Kholy E, Al-Esawy A (2015) Operations on graphs and graph folding. Bulletin of Mathematics and Statistical Research 3: 190-201.

4. Erdos P (1959) Graph Theory and probability. Canadian Mathematical Bulletin 11: 34-38.

5. Harary F (1969) Graph Theory. Addison-Wesley.

6. Haynes TW, Hedetniemi SM, Hedetniemi ST, McCoy TL, Vasylieva L (2012) Cost effective domination in graphs. Congr Numer 211: 197-209.

7. Haynes TW, Hedetniemi ST, Vasylieva I (2015) Very cost effective bipartitions in graphs. AKCE International Journal of Graphs and Combinatorics 12: 155-160.

8. Zeen El-Deen MR, AL-Esawy A. On very cost effective graphs and its folding. 